\title{
Interrelación entre Procedimientos Dinámicos y Cuasi- Estáticos para la Determinación de Propiedades Viscoelásticas de Materiales
}

José D. Melo ${ }^{1}$, Rannier M. Mendonça ${ }^{2}$, Antonio M. Medeiros ${ }^{3}$ y John E.N. Villena ${ }^{4}$ Universidade Federal do Rio Grande do Norte, (1) Departamento de Engenharia de Materiais, (2) Programa de Pós-Graduação em Engenharia Mecânica, (3) Departamento de Engenharia Mecânica, (4) Programa de Pós-Graduação em Ciência e Engenharia de Materiais, Campus Universitário s/n, Lagoa Nova, 59072-970 Natal, RN-Brasil (e-mail: daniel.melo@ufrnet.br)

\begin{abstract}
Resumen
En este trabajo las propiedades viscoelásticas de un polímero fueron determinadas a partir de ensayos cuasi-estáticos y dinámicos, utilizando un equipo de análisis dinámico-mecánico. Se determinó la capacitancia y módulo de relajamiento de muestras de acrilonitrilo butadieno estireno en ensayos de fluencia y de relajamiento de tensión, respectivamente. Ensayos dinámicos con aplicación de excitación armónica fueron también realizados, en un rango de frecuencia definido, y las propiedades medidas fueron utilizadas para calcular la capacitancia y el módulo de relajamiento. La relación entre las propiedades medidas y las calculadas fue evaluada y discutida. Se concluye que existe una buena correlación entre las propiedades medidas y las calculadas.
\end{abstract}

Palabras clave: análisis dinámico-mecánico, viscoelasticidad, fluencia, relajamiento de tensión, polímeros

\section{Interrelation Between Dynamic and Quasi-Static Tests for the Determination Viscoelastic Properties of Materials}

\begin{abstract}
In this investigation, viscoelastic properties of a polymer were determined through quasi-static and dynamic tests, using dynamic mechanical analysis equipment. Creep compliance and relaxation modulus of acrylonitrile butadiene styrene were determined using creep and stress relaxation tests, respectively. In addition, dynamic tests using harmonic excitation were carried out, over a defined range of frequency, and the measured properties were used to calculate creep compliance and relaxation modulus. The relation between measured and calculated properties was evaluated and discussed. The results show good agreement between measured and calculated properties.
\end{abstract}

Keywords: dynamic-mechanical analysis, viscoelasticity, creep, stress relaxation, polymers 


\section{INTRODUCCIÓN}

Muchos materiales presentan comportamiento mecánico dependiente del tiempo, o sea, viscoelasticidad. Este es el caso, por ejemplo, de los polímeros y de los compuestos de matriz polimérica. Las propiedades viscoelásticas de esos materiales son fuertemente dependientes de la temperatura y frecuencia de excitación (Yin et al., 2008; Sarkhel y Choudhury, 2008; Thunga et al., 2008; Setua et al., 2006; Zhang et al., 2006; Tajvidi, 2006; Melo y Radford, 2005). Por lo tanto, el conocimiento de los efectos de temperatura y frecuencia en las propiedades de los materiales es fundamental para el diseño de varios componentes poliméricos.

Polímeros termoplásticos y termofijos son comúnmente estudiados en rangos de frecuencias, cuando se necesita conocer las propiedades de esos materiales en condiciones que representen las condiciones de aplicación (Setua et al., 2006; Melo y Radford, 2005). Comúnmente, los sólidos viscoelásticos tienden a presentar un aumento de módulo de almacenamiento con el aumento de frecuencia (Thunga et al., 2008; Semsarzadeh et al., 2006). La intensidad de ese aumento depende de las transiciones involucradas, pudiendo ser menor en la región vítrea y mayor en el nivel elastomérico. En la región de transición vítrea, la dependencia de frecuencia puede ser aun mayor (Menard, 1999).

Barridos de frecuencia son muy utilizados para la determinación de los efectos de aditivos en las propiedades de un polímero (Semsarzadeh et al., 2006). La utilización de aditivos en polímeros es una práctica común para ajustar las propiedades y reducir los costos. Además, frecuentemente estos aditivos promueven la modificación de propiedades solamente en un determinado rango de frecuencias (Menard, 1999). En estos casos, a través de un ensayo dinámico con barrido de frecuencias, se puede verificar el efecto producido por los aditivos en el rango de frecuencia de utilización del material.

Además de las variaciones de frecuencia y temperatura, propiedades mecánicas de polímeros, son también muy afectadas por variaciones de formulación y otros factores ambientales como la humedad (Yin et al., 2008; Thunga et al., 2008; Gibson, 2000). Se sabe que la humedad puede producir reducción de módulo y aumentar la disipación de energía en polímeros y en compuestos de matriz polimérica (Gibson, 1994; Gibson, 2000). A pesar de que algunos metales también presentan propiedades dependientes de algunos de los factores mencionados, estos son normalmente menos sensibles a esas variaciones. Por lo tanto, el estudio de propiedades mecánicas de polímeros, en las condiciones reales (o próximas) de utilización es fundamental para la aplicación adecuada de esos materiales.

El análisis dinámico-mecánico (DMA), es una técnica ampliamente aceptada por la industria y centros de investigación para caracterización viscoelástica de polímeros. A través de la aplicación de una excitación senoidal, en grandes rangos de frecuencia y temperatura, esta técnica proporciona informaciones importantes sobre la cura de resinas termofijas (Drogar et al., 2008; Worzakowska, 2007) o el proceso de envejecimiento de resinas termoplásticas (Menard, 1999). El método produce resultados de forma rápida y confiable, utilizando cantidades muy pequeñas de material. $A$ través de equipos de análisis dinámico-mecánico, se puede también realizar ensayos de fluencia y de relajamiento de tensión. Ensayos de fluencia y de relajamiento de tensión son las formas mas simples y directas de obtener propiedades mecánicas de materiales relacionadas con la teoría de la viscoelasticidad (Christensen, 2003).

Interrelaciones aproximadas entre propiedades viscoelásticas de materiales obtenidas a partir de ensayos de fluencia y de relajamiento de tensión y de ensayos con excitación armónica, han sido publicadas en la literatura (Christensen, 2003). Se mostró, teóricamente, que es posible establecer relaciones directas entre los componentes real e imaginario del módulo y las funciones de fluencia y de relajamiento de tensión.

Este trabajo tiene como objetivo evaluar la interrelación entre diferentes formas de ensayo para estudiar las propiedades viscoelásticas de polímeros a través de equipos de DMA. Tres cuerpos de prueba de ABS (Acrilonitrilo Butadieno Estireno), con la misma composición química, fueron 
analizados, a través de ensayos de fluencia, de relajamiento de tensión y de ensayos dinámicos con excitación armónica, para determinar la relación entre las propiedades medidas en ambas formas.

\section{RELACIONES ENTRE LAS PROPIEDADES VISCOELÁSTICAS}

El comportamiento viscoelástico lineal de materiales puede ser expresado por las siguientes relaciones constitutivas (Melo, 2002):

$\sigma(t)=\int_{-\infty}^{t} E(t-\tau) \frac{\partial \varepsilon(\tau)}{\partial \tau} d \tau$

0

$\varepsilon(t)=\int_{-\infty}^{t} J(t-\tau) \frac{\partial \sigma(\tau)}{\partial \tau} d \tau$

donde $E(t)$ y $J(t)$ son las funciones de relajamiento de tensión y de fluencia, respectivamente. Se definen $\mathrm{E}(\mathrm{t})$ como el módulo de relajación y $\mathrm{J}(\mathrm{t})$ como capacitancia.

Para un material viscoelástico sometido a una excitación armónica, existe una diferencia de fase entre las tensiones y deformaciones. Considerando una deformación senoidal con frecuencia $\omega$ y amplitud de deformación, $\varepsilon^{0}$, la deformación compleja, $\varepsilon^{\star}$, puede ser expresada como,

$\varepsilon^{*}(t)=\varepsilon^{0} e^{i \omega t}$

En este caso, la ecuación (1) puede ser expresada como,

$\sigma^{*}(t)=\int_{-\infty}^{t} E(t-\tau) \frac{\partial \varepsilon^{*}(\tau)}{\partial \tau} d \tau$

0

$\sigma^{*}(t)=\int_{-\infty}^{t} E(t-\tau) i \omega \varepsilon^{0} e^{i \omega \tau} d \tau$

Definiendo $u=t-\tau \Rightarrow d u=-d \tau$,

$$
\begin{aligned}
& \sigma^{*}=i \omega \varepsilon^{0} \int_{\infty}^{0} E(u) e^{i \omega(t-u)}(-d u) \\
& \sigma^{*}=i \omega \varepsilon^{0} e^{i \omega t} \int_{0}^{\infty} E(u) e^{-i \omega u} d u \\
& \sigma^{*}=i \omega \varepsilon^{*} \int_{0}^{\infty} E(u)(\cos \omega u-i \sin \omega u) d u
\end{aligned}
$$

0

$\sigma^{*}=E^{*} \varepsilon^{*}$

Entonces, el módulo complejo $E^{\star}$ es dado por: 
$E^{*}=i \omega \int_{0}^{\infty} E(u)(\cos \omega u-i \sin \omega u) d u$

$E^{*}=\omega \int_{0}^{\infty} E(u) \sin \omega u d u+i \omega \int_{0}^{\infty} E(u) \cos \omega u d u$

$E^{*}=E^{\prime}+i E^{\prime \prime}$

donde

$E^{\prime}(\omega)=\omega \int_{0}^{\infty} E(u) \sin \omega u d u$

$E^{\prime \prime}(\omega)=\omega \int_{0}^{\infty} E(u) \cos \omega u d u$

E' y E" son denominados módulo de almacenamiento y de pérdida, respectivamente.

La transformada inversa de Fourier puede ser usada para obtener la función de relajamiento de tensión a partir del módulo de almacenamiento o de perdida (Melo, 2002). En ese caso,

$E(t)=\frac{2}{\pi} \int_{0}^{\infty} \frac{E^{\prime}(\omega)}{\omega} \sin \omega t d \omega$

0

$E(t)=\frac{2}{\pi} \int_{0}^{\infty} \frac{E^{\prime \prime}(\omega)}{\omega} \cos \omega t d \omega$

Para determinar una relación directa entre la función de relajamiento de tensión y el módulo de almacenamiento, se hace uso de un artificio matemático (Christensen, 2003). El termino sin $\omega t / \omega$ del integrando es sustituido por una función delta de Dirac,

$\frac{\sin \omega t}{\omega} \rightarrow \frac{\pi}{2} \delta(\omega-\varpi)$

donde $\pi / 2$ corresponde a la integral de $\sin \omega t / \omega$ y $\varpi$ determina la localización de la función delta.

El parámetro $\varpi$ es determinado de forma que la función delta produzca el mismo primer momento en relación al origen que la función $\sin \omega t / \omega$, o sea

$\varpi=\frac{\int_{0}^{\infty} \omega \frac{\sin \omega t}{\omega} d \omega}{\int_{0}^{\infty} \frac{\sin \omega t}{\omega} d \omega}$ 
La solución de la integral resulta en

$\varpi=\frac{2}{\pi t}$

Sustituyendo (12) en (10) y aplicando (14), se encuentra (Christensen, 2003)

$\left.E(t) \approx E^{\prime}(\omega)\right|_{\omega \rightarrow \frac{2}{\pi t}}$

De la misma forma, una relación similar puede ser establecida para la función de fluencia (Christensen, 2003). En ese caso:

$\left.J(t) \approx J^{\prime}(\omega)\right|_{\omega \rightarrow \frac{2}{\pi t}}$

Donde,

$J^{\prime}(\omega)=\frac{E^{\prime}(\omega)}{\left[E^{\prime}(\omega)\right]^{2}+\left[E^{\prime \prime}(\omega)\right]^{2}}$

o sea,

$\left.J(t) \approx \frac{E^{\prime}(\omega)}{\left[E^{\prime}(\omega)\right]^{2}+\left[E^{\prime \prime}(\omega)\right]^{2}}\right|_{\omega \rightarrow \frac{2}{\pi t}}$

De esa forma, la función de relajamiento de tensión y la función de fluencia son determinadas en función del módulo de almacenamiento y de pérdida, por las Ecs. (15) y (18), respectivamente.

\section{PROCEDIMIENTO}

Tres cuerpos de prueba de $A B S$, con la misma composición química, fueron utilizados para los ensayos en el DMA. Las dimensiones nominales finales fueron: $60 \times 12,5 \times 3,5$ mm (C x L x E) (Fig. 1 a). Todos los ensayos fueron realizados en un TA Dynamic Mechanical Analyzer - DMA Q800, en el modo de flexión con vigas engastadas, con $35 \mathrm{~mm}$ entre los apoyos. El DMA Q800 tiene capacidad de realizar ensayos en el rango de frecuencia de 0.01 a $200 \mathrm{~Hz}$ y rango de temperatura de -150 a $600{ }^{\circ} \mathrm{C}$, con resolución de $1 \mathrm{~nm}$ para desplazamiento y fuerza máxima aplicada de $18 \mathrm{~N}$ con resolución de $10^{-5} \mathrm{~N}$. Un torque de $0,678 \mathrm{~N} * \mathrm{~m}\left(6,0 \mathrm{lb}{ }^{*}\right.$ in $)$ fue aplicado en los puntos de anclaje del cuerpo de prueba (Fig. 1 b). Los cuerpos de prueba utilizados fueron proporcionados por la TA Instruments para fines de calibración del equipo.

Inicialmente los ensayos de fluencia fueron realizados en un cuerpo de prueba, en varios niveles de tensión, para determinar la región viscoelástica linear del material. Los ensayos fueron realizados en un intervalo de tiempo de 1,0 min, las deformaciones en un tiempo de 0,5 min, fueron comparadas para los diversos niveles de tensión. Todos los ensayos fueron realizados a temperatura ambiente $\left(\sim 30^{\circ} \mathrm{C}\right)$. A partir de los datos de estos ensayos, se verificó que una tensión de 1,0 MPa podría ser 
aplicada, correspondiendo a una deformación de aproximadamente 0,05\%, con el material permaneciendo en el régimen viscoelástico lineal.
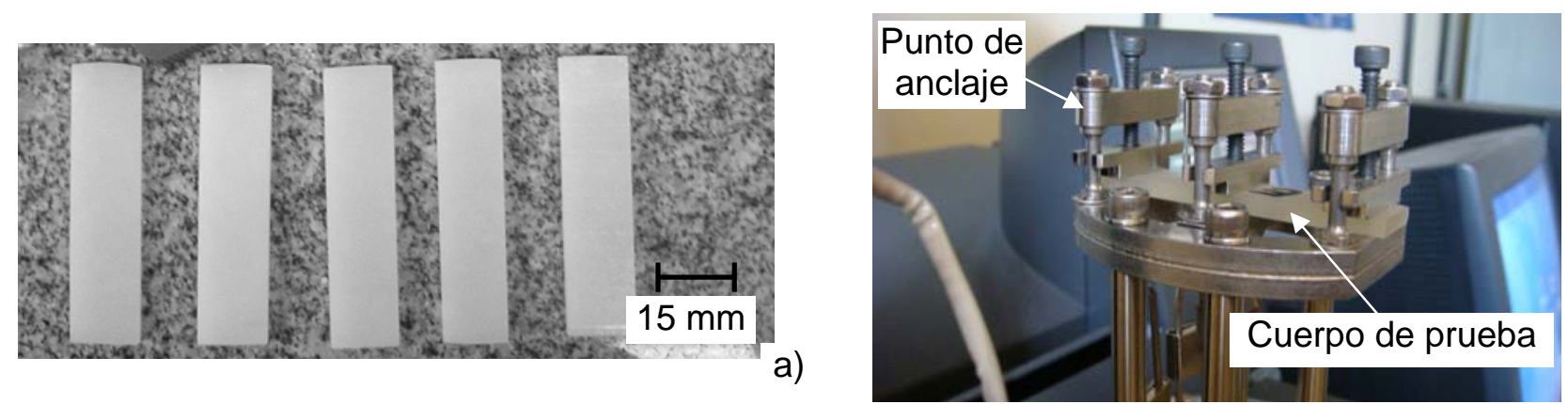

b)

Fig. 1: a) Cuerpos de prueba de ABS para ensayos de flexión en el DMA. b) Esquema de montaje experimental.

Los ensayos dinámico-mecánicos fueron realizados con control de deformación, en un rango de frecuencia de $0,01 \mathrm{~Hz}$ a $130 \mathrm{~Hz}$. De esa manera, datos de parámetros viscoelásticos fueron obtenidos para frecuencias de hasta $100 \mathrm{~Hz}$. La frecuencia de $0,01 \mathrm{~Hz}$ representa el límite inferior del rango de frecuencia disponible en el equipo. Una amplitud de deformación máxima de 0,05\% fue aplicada durante los ensayos.

Los ensayos de fluencia fueron realizados en un intervalo de tiempo de $30 \mathrm{~s}$. El equipo fue programado para obtener datos a una razón de 0,1 s/pt, que representa la tasa máxima disponible en el equipo. Para esos ensayos de fluencia, se aplicó una tensión constante de 1,0 MPa, que resultó en una deformación inicial aproximada de 0,05 \%. La capacitancia de fluencia fue medida en función del tiempo.

Los ensayos de relajamiento de tensión fueron realizados aplicando una deformación constante de $0,05 \%$ y midiendo la tensión a una tasa de 0,1 s/pt. La función de relajamiento fue determinada para un intervalo de tiempo de $20 \mathrm{~s}$.

\section{RESULTADOS Y DISCUSIÓN}

El resultado de los ensayos de fluencia para la determinación de la región viscoelástica lineal del polímero (ABS) se presenta en la Fig. 2, en donde se verifica un incremento proporcional en la deformación con el aumento de la tensión. Estos ensayos, indican que la tensión de 1 MPa, que corresponde a una deformación de 0,05\%, aproximadamente, en un intervalo de tiempo de 0,5 min, fue definida para la realización de los ensayos de fluencia. Para los ensayos dinámicos con barrido de frecuencia fue utilizada una amplitud de deformación de 0,05 \%, y este mismo valor fue aplicado como deformación constante para los ensayos de relajamiento de tensión.

Las propiedades viscoelásticas (módulo de almacenamiento y de pérdida) de tres cuerpos de prueba del polímero determinadas en los ensayos dinámicos con excitación senoidal y en función de la frecuencia, son mostradas en las Figs. 3(a) y 3(b), respectivamente.

Las medidas del módulo de almacenamiento presentaron una buena repetitividad entre los tres cuerpos de prueba ensayados (Fig. 3). Se observa una tendencia de aumento del módulo de almacenamiento con el aumento de la frecuencia mostrado en escala logarítmica. Para las curvas de módulo de pérdida, se percibe un desvió mayor en la fase inicial del ensayo para una de las muestras (ABS 03). Pero, esa variación del módulo de pérdida no interfiere de forma significativa en los cálculos de capacitancia (función de fluencia), ecuación (18), tomando en cuenta que su valor es muy pequeño en relación al módulo de almacenamiento. El módulo de pérdida, para el cálculo del módulo de relajamiento (ecuación (15)), no es considerado. 


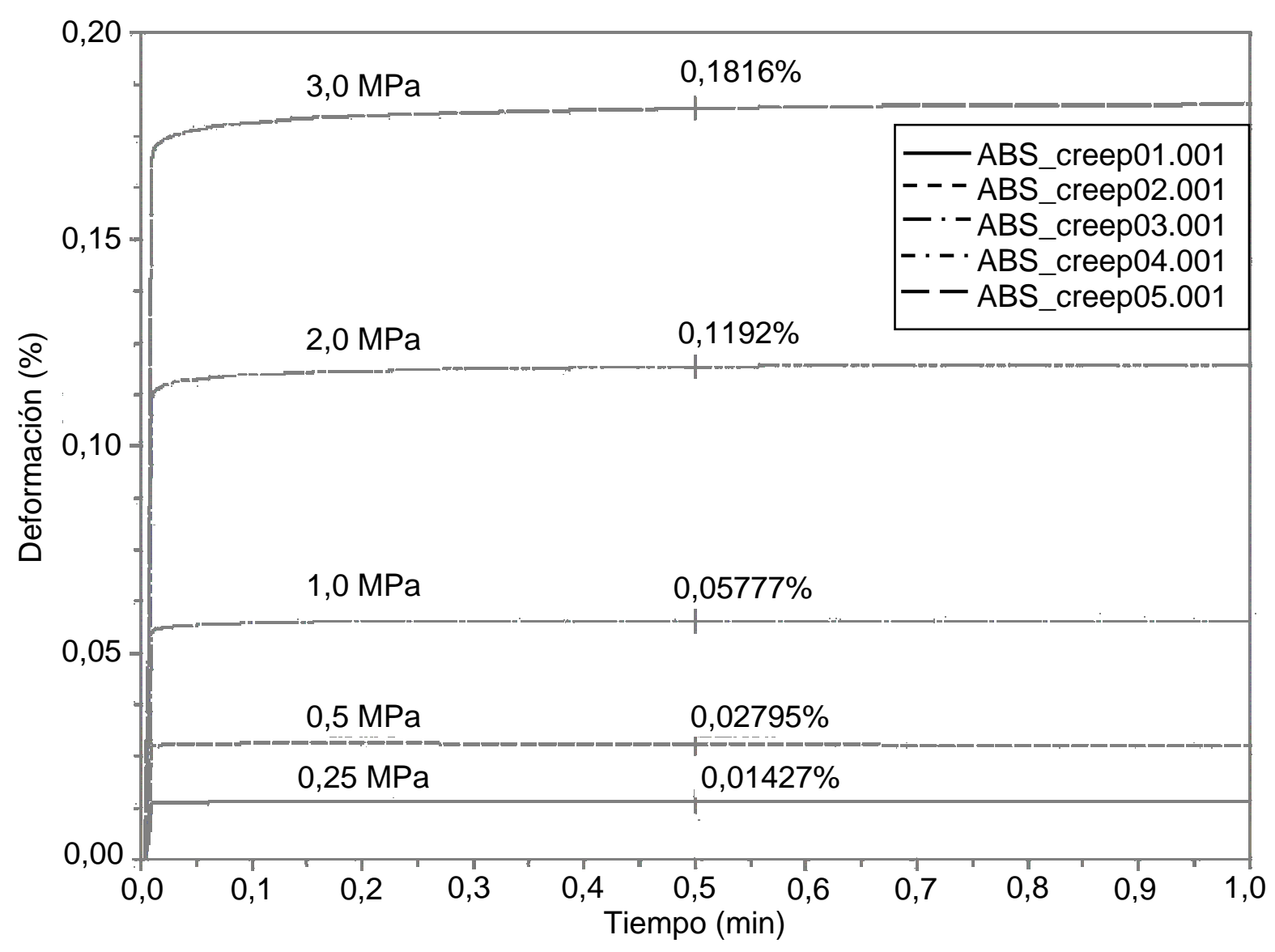

Fig. 2: Ensayos de fluencia para determinación de la región viscoelástica lineal de ABS.

En las Figs. 4 y 5 son mostrados los resultados de los ensayos de fluencia y de relajamiento de tensión, respectivamente, correspondientes a tres muestras. En los dos casos, se verifica una variación inicial de las propiedades (capacitancia y módulo de relajamiento), respectivamente. Esta variación inicial corresponde al proceso de aplicación de la solicitación inicial, o sea, tensión, para los ensayos de fluencia, y deformación, para los ensayos de relajamiento de tensión. Con el equipo (DMA) utilizado en los experimentos, el tiempo de aplicación de la solicitación inicial fue de aproximadamente 0,6 s (0,01 min), para los ensayos de fluencia, y de 4,8 s (0,08 min), para los ensayos de relajamiento de tensión.

Las propiedades viscoelásticas, módulos de almacenamiento y de pérdida, determinados en ensayos dinámicos (Fig. 3 (a) y (b)), en función de la frecuencia, fueron utilizadas para calcular la capacitancia y el módulo de relajamiento, en función del tiempo, de acuerdo con las Ecs. (18) y (15), respectivamente, para fines de comparación con los valores de capacitancia y módulo de relajamiento medidos (Figs. 4 y 5). Así, para los valores iniciales calculados de capacitancia y módulo de relajamiento, se utilizaron valores módulos de almacenamiento y de pérdida medidos con una frecuencia de $100 \mathrm{~Hz}$, que corresponden a un tiempo de 10-3 s, para sustitución en las Ecs. (15) y (18). Estos valores fueron comparados con los valores iniciales de capacitancia y módulo de relajamiento, medidos inmediatamente después de la aplicación completa de la solicitación inicial, o sea, $t=0,6 \mathrm{~s}$ para los ensayos de fluencia, y $t=4,8 \mathrm{~s}$ (0,08 $\mathrm{min})$, para los ensayos de relajamiento de tensión. De la misma manera, las propiedades medidas en ensayos dinámicos con una frecuencia de $0,01 \mathrm{~Hz}$, fueron comparadas con las determinadas en ensayos de fluencia y de relajamiento de tensión, después de un tiempo de 10,13 s, de la completa aplicación de la solicitación inicial.

La comparación entre la capacitancia medida en ensayos de fluencia y la calculada, utilizando la Ec. (18), a partir de los módulos de almacenamiento y de pérdida, medidos en los ensayos dinámicomecánicos, está mostrada en la Fig. 6, para las tres muestras de ABS. 


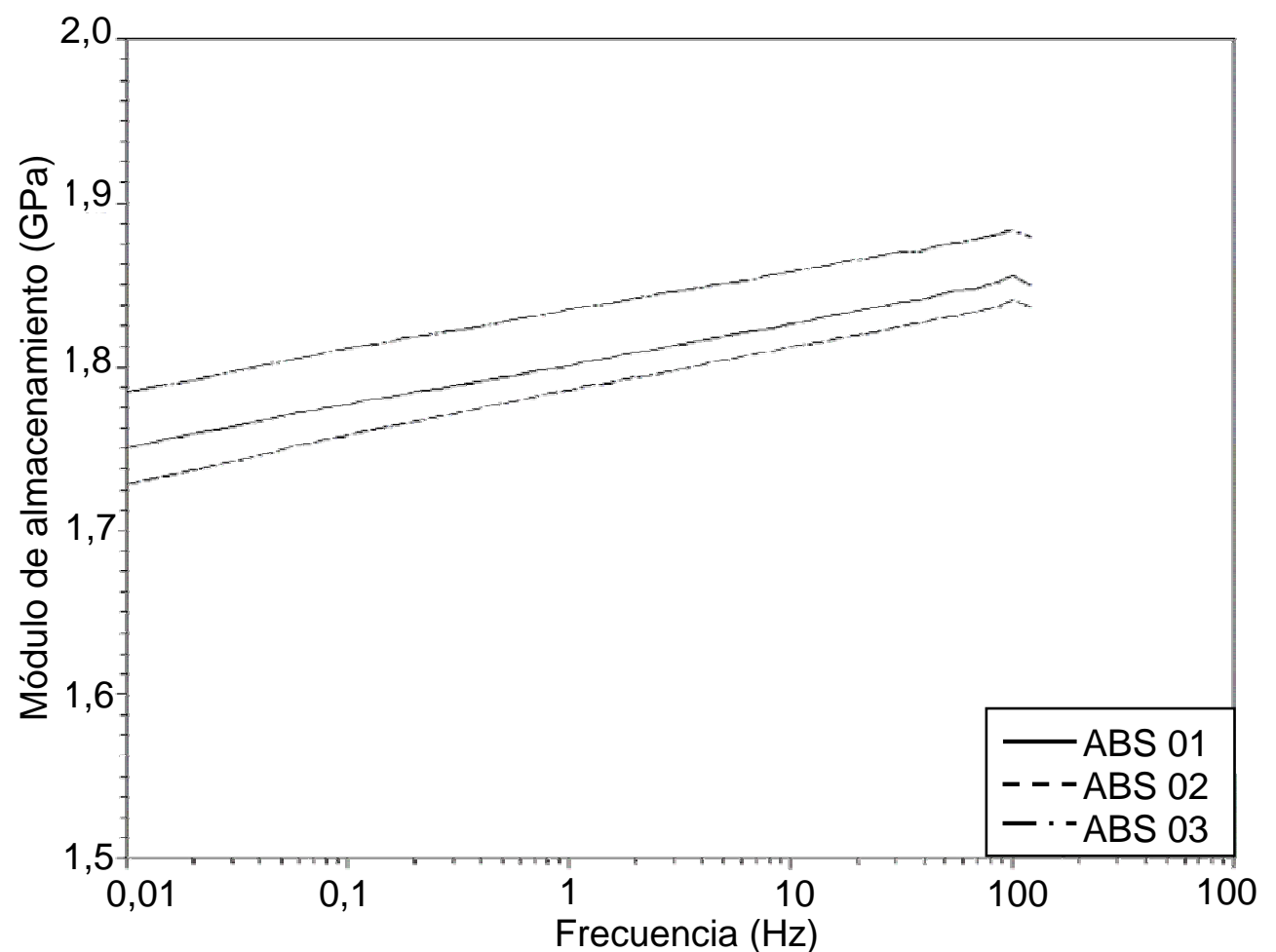

a)

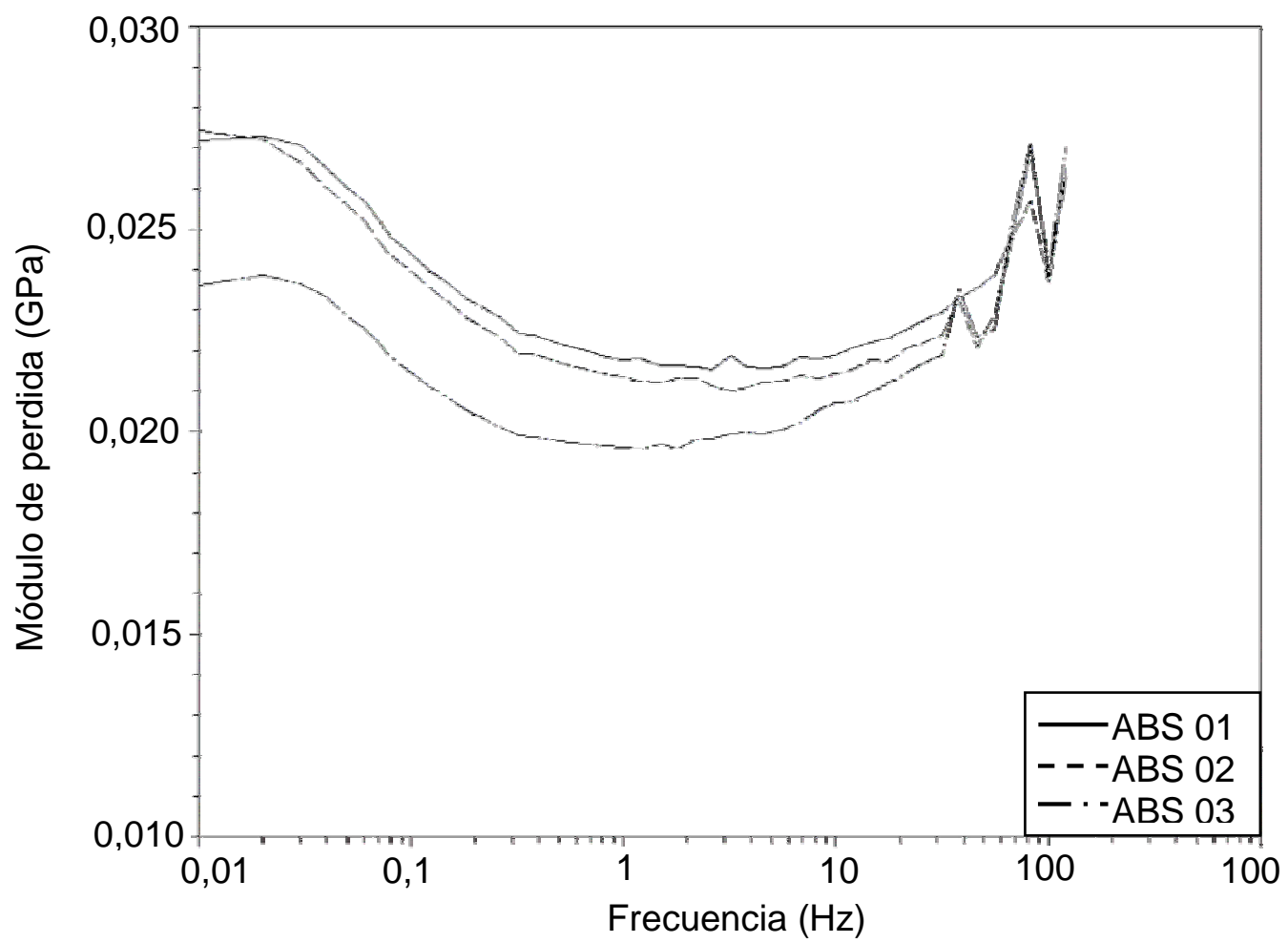

b)

Fig. 3: Propiedades viscoelásticas del ABS en función de la frecuencia. (a) Módulo de almacenamiento y (b) Módulo de pérdida.

Se observa que la curva de capacitancia calculada a partir de los datos de los ensayos dinámicos de barrido de frecuencia (Ec. (18)), presenta valores muy próximos de la curva medida por el equipo (DMA) en ensayos de fluencia (Fig. 6). Luego, la Ec. (18) mostró ser válida para los datos experimentales obtenidos en este estudio. Pero, en virtud de limitaciones del equipo utilizado, el intervalo de tiempo donde fue realizada la comparación entre la capacitancia medida (ensayos de fluencia) y la calculada (Ec. (18)) es bastante reducido (aproximadamente $10 \mathrm{~s}$ ). Para calcular la capacitancia en tiempos mayores que $10 \mathrm{~s}$ son necesarios ensayos dinámicos en frecuencias 
menores que $0,01 \mathrm{~Hz}$, lo que sobrepasaría el límite mínimo de frecuencia del equipo $(0,01 \mathrm{~Hz})$. Por otro lado, para comparar valores de capacitancia calculados a través de frecuencias mayores que $100 \mathrm{~Hz}$, son necesarios ensayos de fluencia en tiempos menores que $10^{-3} \mathrm{~s}$. Pero, el equipo necesita de un tiempo mayor $(0,6 \mathrm{~s})$ solamente para aplicar completamente la carga en el ensayo de fluencia (Fig. 6). Además, generalmente, informaciones de fluencia para grandes intervalos de tiempo son de interés.

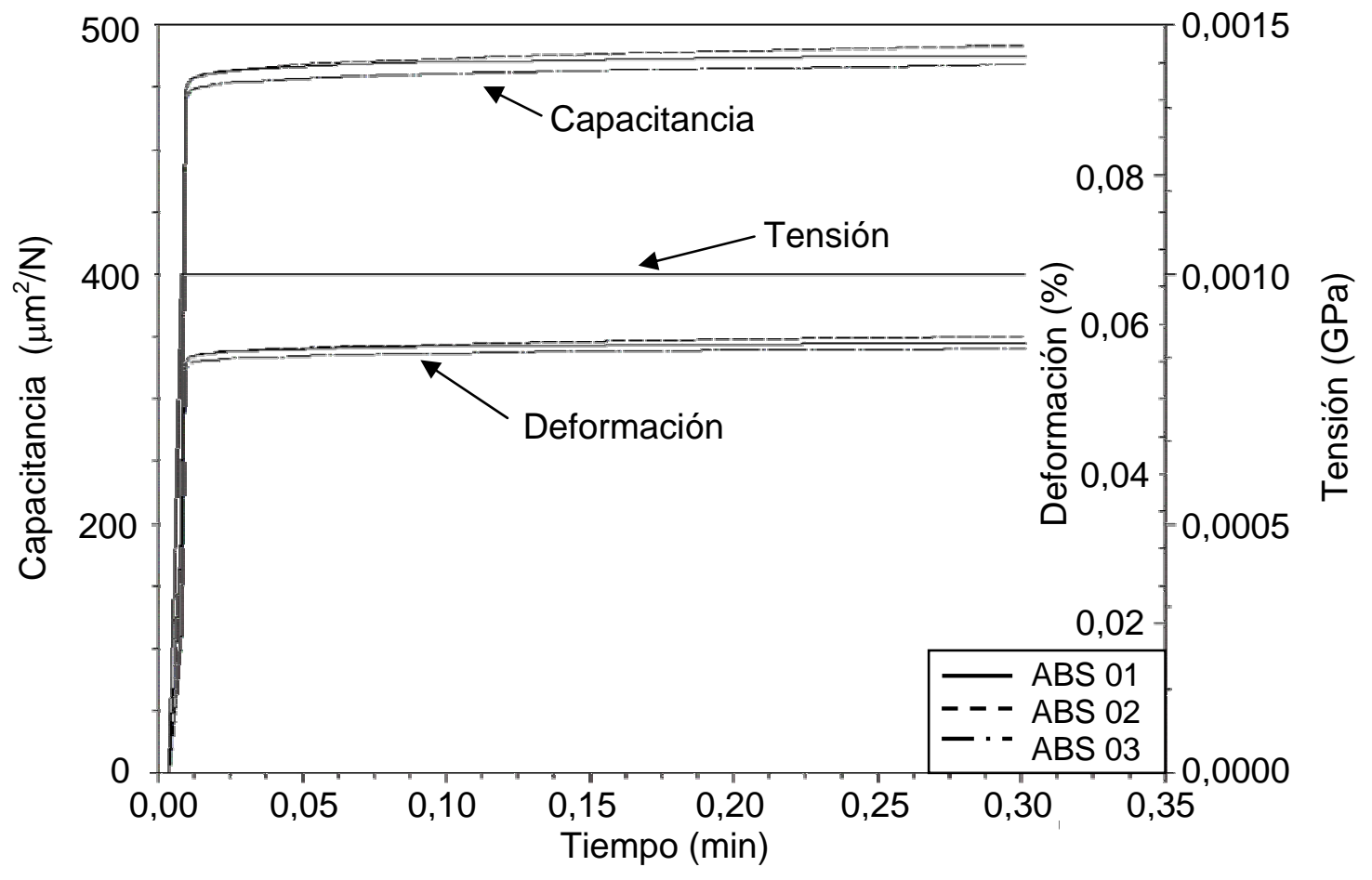

Fig. 4: Capacitancia de muestras de ABS (Ensayo de fluencia).

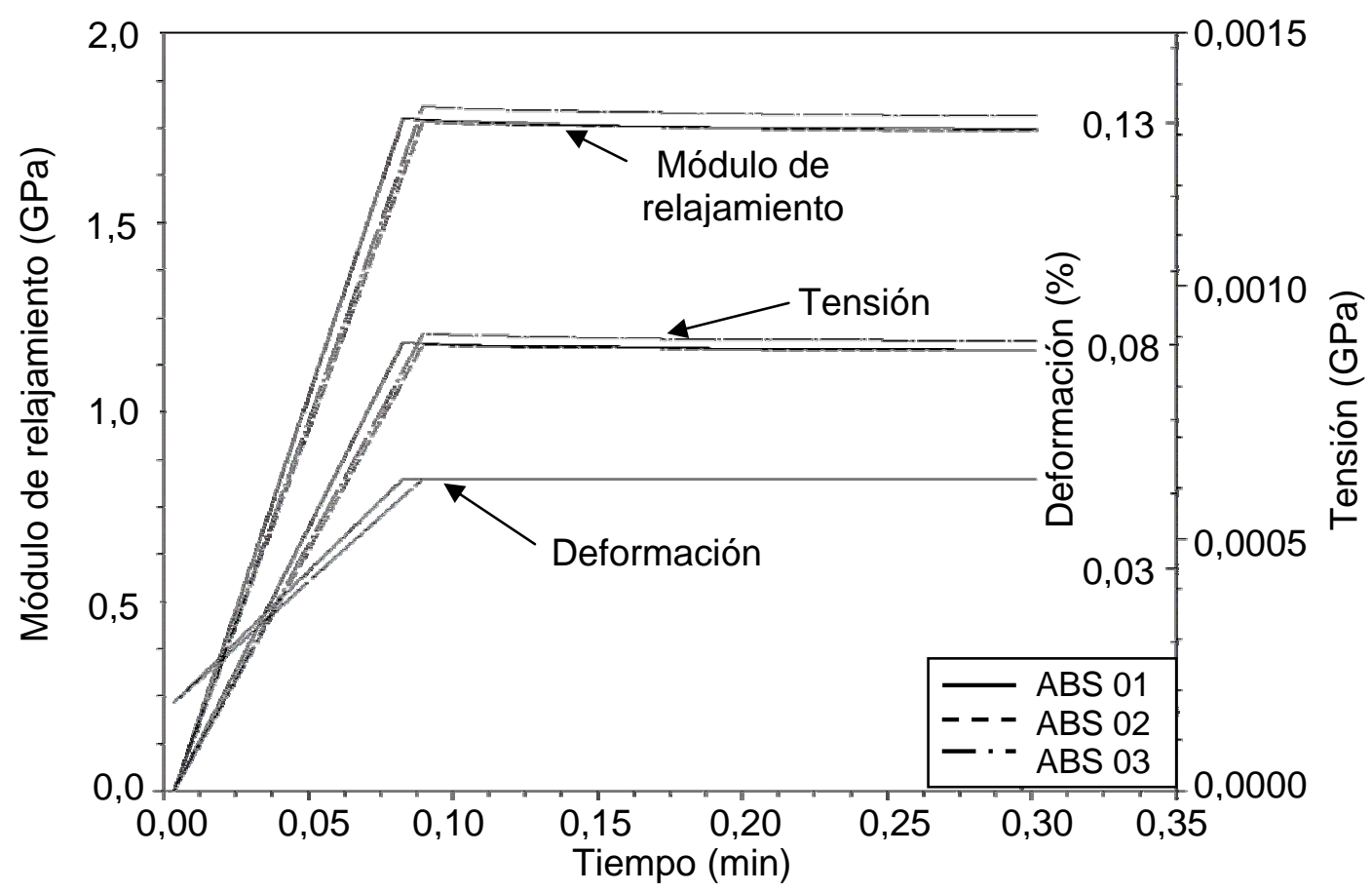

Fig. 5: Módulo de relajamiento de muestras de ABS (Ensayo de relajamiento de tensión). 


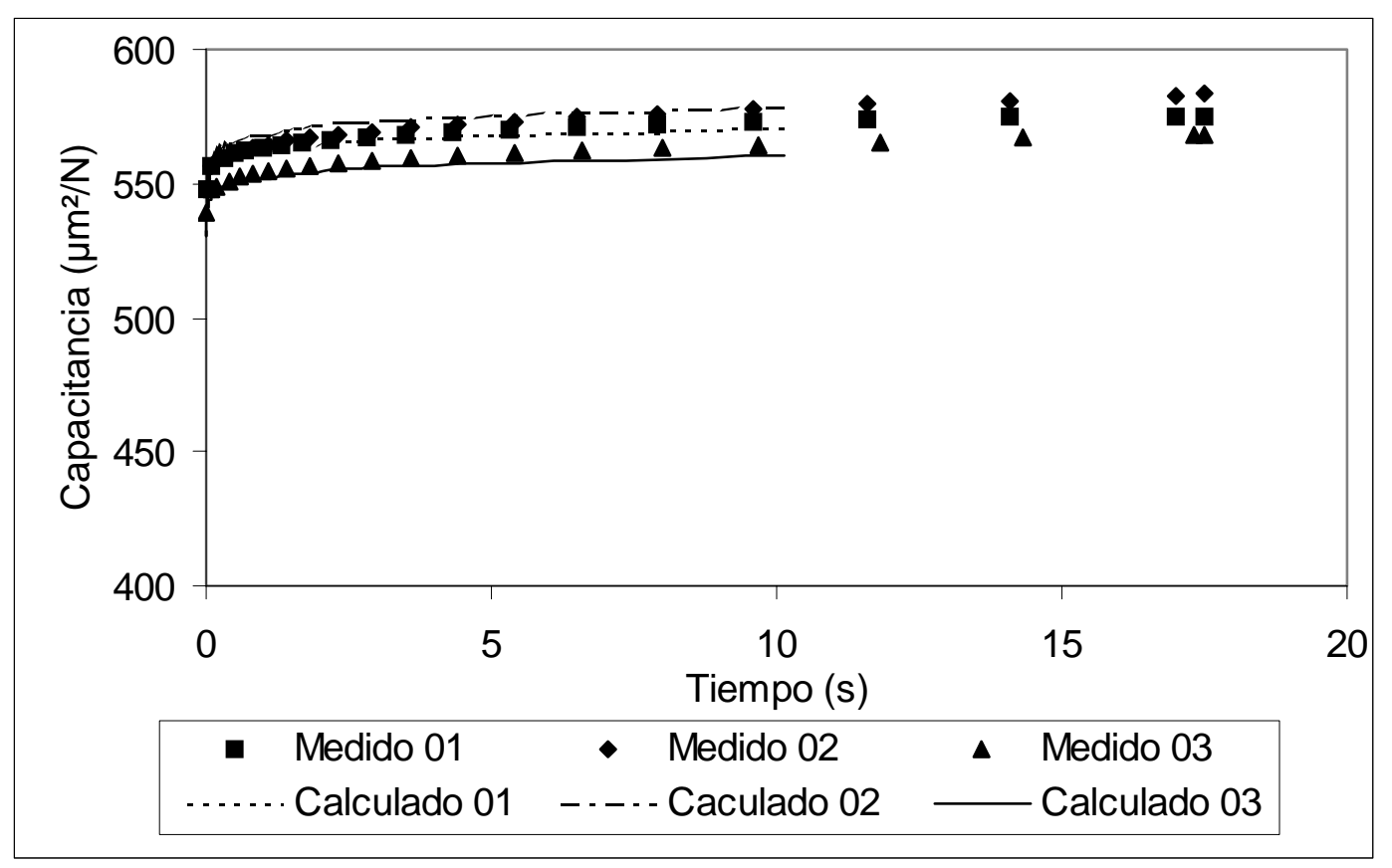

Fig. 6: Relación de los datos de fluencia medidos y calculados.

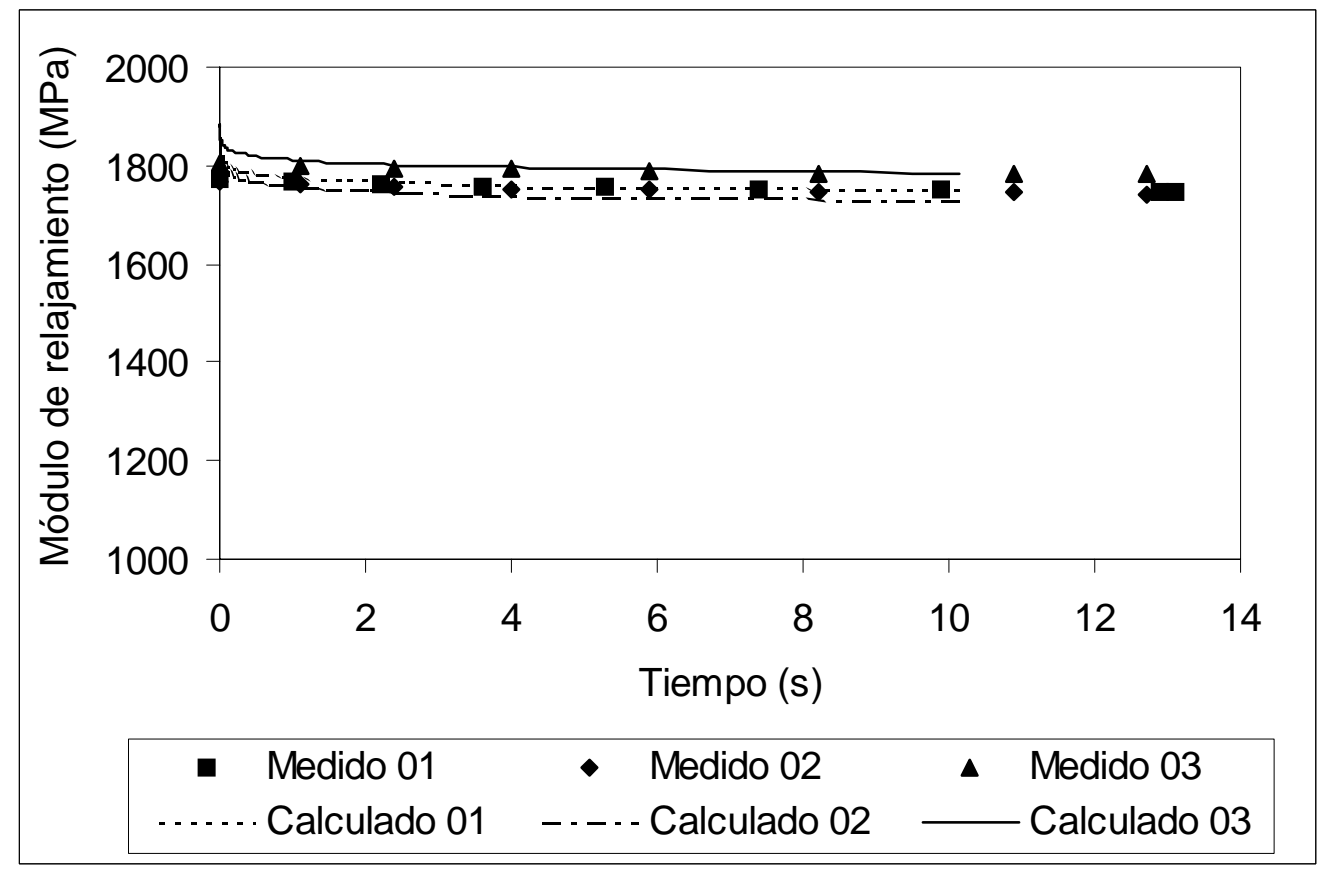

Fig. 7: Relación de los dados de módulo de relajamiento medidos y calculados de cada muestra.

Un procedimiento similar fue realizado para comparar el módulo de relajamiento medido en ensayos de relajamiento de tensión y el calculado a partir del módulo de almacenamiento, Ec. (15) (Fig. 7). La buena correlación entre las propiedades calculadas a partir de los ensayos dinámicos (Ec. (15)) y las medidas en ensayos cuasi-estáticos de relajamiento de tensión puede ser observada. Las mismas limitaciones relativas al intervalo de tiempo (o de frecuencia) ya comentadas para la capacitancia también se aplican para el módulo de relajamiento. Para intervalos de tiempo mayores que $10 \mathrm{~s}$ se necesita frecuencias menores que $0,01 \mathrm{~Hz}$, mientras que, para frecuencias mayores que $100 \mathrm{~Hz}$ se necesita tiempos de aplicación de deformación menores que 10-3 s. Las funciones de relajamiento de tensión y de fluencia determinadas de acuerdo con las Ec. (15) y (18), asumen que los efectos 
transientes pueden ser depreciados. Esto limita los datos de relajamiento de tensión y de fluencia para tiempos mayores que 0,01 s (Christensen, 2003).

Por lo tanto, a través de los datos presentados en este estudio, se verificó una buena correlación entre las propiedades viscoelásticas medidas en ensayos dinámicos y en ensayos de fluencia y de relajamiento de tensión. Además, el intervalo de tiempo (o frecuencia) estudiado quedó bastante restringido a las limitaciones de la técnica experimental utilizada. Para obtener propiedades de los materiales viscoelásticos en un intervalo de tiempo mayor (o frecuencia), se utiliza métodos de superposición de datos como el de superposición de tiempo y temperatura. Este principio puede ser aplicado para materiales que presentan comportamiento termo-reológico simple. En este caso, las propiedades determinadas con base en alguno de los ensayos realizados en este trabajo - dinámico, fluencia o relajamiento de tensión - y en varias temperaturas, son superpuestas para formar una curva maestra. Con la superposición, la curva maestra resultante cubre un intervalo de tiempo (o frecuencia) mucho mayor que los datos obtenidos.

\section{CONCLUSIONES}

Este se investigó la interrelación entre procedimientos dinámicos y cuasi-estáticos para la determinación de propiedades viscoelásticas de los materiales. Capacitancia y módulo de relajamiento de muestras de ABS fueron determinados en ensayos de fluencia y de relajamiento de tensión, respectivamente. Ensayos dinámicos con aplicación de excitación armónica fueron también realizados, en un rango de frecuencia, y las propiedades medidas fueron utilizadas para calcular la capacitancia y el módulo de relajamiento. Se verificó una buena correlación entre las propiedades medidas y las calculadas. Por otro lado, fue demostrado que el intervalo de tiempo (o frecuencia) para este tipo de estudio es bastante restringido, debido a las limitaciones de la técnica y del equipo utilizado.

\section{NOMENCLATURA}

$\begin{array}{ll}\omega & \text { Frecuencia } \\ \sigma & \text { Tensión } \\ \varepsilon & \text { Deformación } \\ \varepsilon^{\star} & \text { Deformación compleja } \\ \sigma^{\star} & \text { Tensión compleja } \\ \varepsilon^{0} & \text { Amplitud de deformación } \\ E(t) & \text { Módulo de relajación } \\ E^{\star} & \text { Módulo complejo } \\ E^{\prime} & \text { Módulo de almacenamiento } \\ E^{\prime \prime} & \text { Módulo de pérdida } \\ J(t) & \text { Capacitancia }\end{array}$

\section{REFERENCIAS}

Christensen, R. M., Theory of Viscoelasticity, 1 Ed, 205 pp. Dover Publications Inc., Mineola, NY, USA (2003).

Droger N.; O. Primel y J.L. Halary; Characterization of the Viscoelastic and Mechanical Properties of Tightly Cross-Linked Polythiourethane Networks, Journal of Applied Polymer Science: 107, 455-462 (2008).

Gibson, R.F.; Principles of Composite Material Mechanics, 1 Ed, 423 pp. New York, NY, McGraw-Hill, (1994).

Gibson, R.F.; Sensitivity of Stiffness and Damping to Various Aspects of Material and Structural Behavior, NCA - Vol.27, Proceedings of the ASME Noise Control and Acoustics Division - 2000, ASME 2000: 301-318 (2000). 
Melo, J.D.D. y D.W. Radford; Time and Temperature Dependence of the Viscoelastic Properties of CFRP by Dynamic Mechanical Analysis. Composite Structures: 70, 240-253 (2005).

Melo, J.D.D.; Elastic and Viscoelastic Characterization of Transversely Isotropic Composite Laminae, $\mathrm{PhD}$. Dissertation, Colorado State University, Department of Mechanical Engineering, Fort Collins, CO, USA (2002).

Menard, K.P.; Dynamic Mechanical Analysis - A Practical Introduction, 2 Ed, 364 pp. CRC Press LLC, Boca Raton, FL, USA, (1999).

Sarkhel G. y A. Choudhury; Dynamic Mechanical and Thermal Properties of PE-EPDM Based Jute Fiber Composites, Journal of Applied Polymer Science: 108, 3442-3453 (2008).

Semsarzadeh, M.A.; S.M. Barikani y M. Ansari; Effect of Temperature on Dynamic Physical Behavior of Poly(vinyl chloride) Gel Structure with Ester Plasticizers, Macromolecular Symposia: 239, 251-258 (2006)

Setua D. K. y otros cinco autores; Determination of Dynamic Mechanical Properties of Engineering Thermoplastics at Wide Frequency Range using Havriliak-Negami Model, Journal of Applied Polymer Science: 100, 677-683 (2006).

Tajvidi M., R.H. Falk y J.C. Hermanson; Effect of Natural Fibers on Thermal and Mechanical Properties of Natural Fiber Polypropylene Composites Studied by Dynamic Mechanical Analysis, Journal of Applied Polymer Science: 101, 4341-4349 (2006).

Thunga M. y otros seis autores; Dynamic Mechanical and Rheological Properties of Binary S-(S/B)-S Triblock Copolymer Blends, Journal of Polymer Science: Part B: Polymer Physics: 46, 329-343 (2008).

Worzakowska M.; Influence of Cure Schedule on the Viscoelastic Properties and Thermal Degradation of Crosslinked Monoand Diepoxides Obtained During the Reaction of Hydrogen Peroxide and Divinylbenzene, Journal of Applied Polymer Science: 103, 462-469 (2007).

Yin Z.N., L.F. Fan y T.J. Wang; Experimental investigation of the viscoelastic deformation of PC, ABS and PC/ABS alloys, Materials Letters: 62, 2750-2753 (2008).

Zhang C., P. Wang, C-A. Ma y M. Sumita; Damping Properties of Chlorinated Polyethylene-Based Hybrids: Effect of Organic Additives, Journal of Applied Polymer Science: 100, 3307-3311 (2006). 